\title{
CIENCIA FICCIÓN CUÁNTICA Y LA ONTOLOGÍA DEL CONJUNTO VACÍO
}

\section{QUANTUM SCIENCE FICTION AND THE ONTOLOGY OF THE EMPTY SET}

Roy Alfaro Vargas ${ }^{1}$

Investigador independiente, Costa Rica.

royalfarov@gmail.com

Recibido: 22 de marzo del 2017 / Aprobado: 6 de setiembre del 2017

Publicado: 7 de noviembre de 2017

\section{Resumen:}

Este artículo analiza la relación entre la ciencia ficción y la ontología de la teoría de conjuntos, como un medio de acceder a algunos procesos diegéticos del cine de este género, y su conexión con algunos fenómenos sociales y económicos. Además, se establece un vínculo entre la que se denomina aquí ciencia ficción cuántica, la teoría de cuerdas y las propuestas de John Rieder, ligadas a la implementación estética de la ontología del conjunto vacío, en el contexto de algunas ideas desarrolladas por Alain Badiou, en este tipo de producción cultural.

Palabras clave: filosofía, estudios culturales marxistas, novum, física, neoliberalismo.

\section{Summary:}

This article analyzes the relation between science fiction and the ontology of set theory, as a means to access to some diegetic process of science fiction genre and its connection with some social and economic phenomena. Also, it is set up a link among the here so-called quantum science fiction, string theory and the proposals by John Rieder, linked to the aesthetic implementation of the ontology of void set, in the context of some ideas developed by Alain Badiou, into this kind of cultural production.

Keywords: philosophy, marxist cultural studies, novum, physics, neoliberalism.

1 Bachiller en la Enseñanza de la Literatura y el Castellano. Egresado de la Maestría en Literatura Latinoamericana, Universidad de Costa Rica. 


\section{Introducción}

Se aborda en este escrito la ciencia ficción (CF) en relación con el desarrollo de algunas propuestas en física (la teoría de cuerdas, específicamente), así como con el fundamento ontoepistemológico de esta, o sea, la teoría de conjuntos. Asimismo, la discusión sobre la CF aquí analizada se inserta en procesos sociales como el neoliberalismo y el postpostmodernismo, sin olvidar el concepto de novum tecnocrático.

La tesis que nos guiará es la siguiente: en el fenómeno del novum tecnocrático, existe un segmento de la actual CF que denominaremos CF cuántica, caracterizada por ligarse a los planteamientos de algunas teorías físicas, como la teoría de cuerdas, teniendo como fundamento ontoepistemológico la teoría de conjuntos; en ella la función de la CF cuántica es elaborar una escatología no-teológica y promover, en algunos casos, una concepción voluntarista.

Para probar esta tesis es preciso definir el concepto de novum tecnocrático, así como caracterizar la teoría de cuerdas y algunos elementos de la teoría de conjuntos, sin olvidar lo que vamos a entender como CF cuántica. Asimismo, va a ser necesario determinar el funcionamiento de los esquemas de la CF cuántica en algunas películas recientes, con el fin de señalar las funciones ideológicas de esta CF cuántica.

\section{El concepto de novum tecnocrático}

La noción de novum tecnocrático permite explicar, formal y socialmente, gran parte de las actuales producciones de CF. Este se define, entonces, como un extrañamiento no-cognitivo, que extraña por no hacer referencia a la realidad y que, por tanto, no implica un proceso cognitivo, su función es crear un velo ideológico sobre el contexto de origen (Alfaro-Vargas, 2015).

Ser una noción formal es lo que define al novum tecnocrático, su contenido extracinematográfico o extraliterario es absorbido por el efecto de espectacularidad (Alfaro-Vargas, 2015), con un marcado "énfasis en lo emocional en detrimento de lo racional” (Alfaro-Vargas, 2015, p. 13). Este énfasis sitúa la CF nóvico-tecnocrática en la dictadura de la emoción (Han, 2014), que "impulsa la emocionalización del proceso productivo" (Han, 2014, p. 72), en tanto "El régimen neoliberal presupone las emociones 
como recursos para incrementar la productividad y el rendimiento" (Han, 2014, p. 71), así como la instrumentalización de las emociones como recurso ideológico. Atestiguamos así la expansión de una visión cultural entendida como cultura emocional, la cual se define como "el conjunto de significados y códigos operativos (o 'técnicas') por medio de los cuales la gente maneja, despliega y comprende sus propias emociones y acciones" (García-Martínez y González, 2016, p. 14)². Esta es una cultura invadida por la emoción, en tanto componente no-racional, que permea tanto la vida cotidiana como la academia ${ }^{3}$, en un proceso que implica el paso "del discurso emocional desde la psicología hasta una multitud de esferas sociales, eventualmente, emergiendo dentro de la cultura popular a través de los medios de masas" (García-Martínez \& González, 2016, p. 15) y de la CF como medio de masas que es.

La función estética de la formalización, que está implícita en el novum tecnocrático, oculta el verdadero contenido de este, o sea, su rol como elemento ideológico-pedagógico. Es decir, el novum tecnocrático, aparte de ser "una forma de evasión, de escapismo" (Alfaro-Vargas, 2015, p. 15), gira "alrededor de la necesidad de promover la ciencia y la matemática" (Alfaro-Vargas, 2015, p. 16) como un medio más para reavivar la economía capitalista, en crisis desde los años 1970. Así, este novum cumple ideológicamente una doble y contradictoria función, o sea, se pretende una CF que estimule el desarrollo científico-tecnológico y, al mismo tiempo, una CF que permita el control social (Alfaro-Vargas, 2014) a través del énfasis en lo irracional y en la emoción.

Por ende, la CF ligada al novum tecnocrático es un producto cultural que, a lo postpostmoderno, cree ingenuamente que puede definirse como $\mathrm{A}$ y no-A al mismo tiempo. La fantasización de la CF implica crear un relato que "puede contener imposibilidades lógicas" (Alber, 2016, p. 30), donde irracionalmente " $p$ y non-p son simultáneamente verdaderos" (Alber et al. 2013, p. 105). Este novum se pretende estimulador de la inteligencia y

2 Las traducciones de textos citados del inglés, francés, portugués y alemán son del autor.

3 Ejemplo de esta emocionalización de la academia es el artículo mismo de Saldías (2016), motivo por el cual él no puede entender que "lo que cuenta como ficción es lo que las instituciones dominantes llaman ficción" (Stock, 2016, p. 211) y que hoy las instituciones neoliberales le llaman CF a esta manipulación ideológico-económica que se ha denominado el novum tecnocrático: la CF "es usada como un medio de adoctrinamiento" (May, 2017, p. x). 
mecanismo de estupidización (como control social), incentivando el pensamiento científico lógico-racional con cuentos de hadas (post)postmodernos. Tal novum sirve para socializar "valores ligados tanto al neoliberalismo, como de su ideología (post) postmoderna” (Alfaro-Vargas, 2015, p. 18).

\section{La CF cuántica: características y contexto}

Llamaremos CF cuántica a aquellas producciones de CF ancladas en el novum tecnocrático, pero que tienen como eje específico en su estructuración diegética elementos de la mecánica cuántica y la relatividad especial (general), o sea la teoría de cuerdas, mediante la representación de escenarios relacionados con universos paralelos o dimensiones alternas.

No obstante, si bien una definición inicial es útil para el proceso comunicativo entre escritor y lector, lo cierto es que es necesario profundizar la cuestión emparentada con algunos elementos de física y su ligamen con la CF, alrededor de la teoría de conjuntos.

Iniciemos con Lacan, para comprender desde dónde se abordará la relación entre CF, física (teoría de cuerdas) y la teoría de conjuntos. Lacan introduce la categoría de conjunto como un medio para deshacerse de la noción de totalidad: "La categoría de conjunto, para introducirlo, encuentra nuestra aprobación, en tanto que evita las implicaciones de la totalidad o las elimina" (Lacan, 1966, p. 648). Con esto, Lacan elimina el contenido real, material, concreto y multi determinado del ser como movimiento; como cambio, para quedarse con un cascarón formal, que es el conjunto vacío $(\varnothing)$.

\section{El concepto de conjunto y de conjunto vacío $(\emptyset)$ : una ontoepistemología}

Alfaro-Vargas (2016), en “Totalidad y teoría de conjuntos” ${ }^{4}$ explica el rol del conjunto vacío en el contexto actual, donde las propuestas de Alain Badiou son fundamentales.

De este modo, Alfaro-Vargas (2016) define un conjunto como una colección de elementos. Por ejemplo, podemos plantear un conjunto $X$, que contiene los elementos a, b, c; es decir $X=\{a, b, c\}$. Asimismo, se señala, entre los lineamientos de la teoría de conjuntos, que el fundamento de todo

4 Este artículo nos dará entonces la base para abordar nuestra tesis. 
conjunto es el conjunto $\varnothing$, ya que el axioma de existencia reza que "hay un conjunto (y este es el conjunto vacío)" (Gironi, 2015, p. 24), el cual existe como tal, pero no implica una extensión, un grupo de objetos. La asunción del conjunto $\varnothing$ funda, por tanto, una lógica intensional, que, al eliminar la extensionalidad (la referencialidad) del conjunto $\varnothing$, privilegia la forma sobre el contenido, así como la parte sobre la totalidad, al asumir el conjunto $\varnothing$ como la base ontoepistemológica que plantea que "el ser es inmóvil" (Badiou, 2006, p. 378). Esto lleva a "una disolución de la materia" (Burhanuddin, 2015, p. 12), o sea, esta es una ontología desprovista de contenido empírico, sin extensión y con una intensión lógica nula.

Con este logicismo matemático, se olvida que la totalidad es indispensable (Lefebvre, 2016) y, en cambio, nos lleva por el camino del solipsismo y del nihilismo (Alfaro-Vargas, 2016). Esta perspectiva de la teoría de conjuntos nos deja un lenguaje matemático (dependiente del natural) que crea realidades, es decir, modelos. Y es desde este establecimiento de modelos que se producen teorías "físicas", como la teoría de cuerdas, así como una parte de la CF actual, que Rieder (2010) trata de justificar con el esquema de modelos.

\section{La teoría de cuerdas}

Desde el siglo pasado, en relación con la teoría general de la relatividad y la mecánica cuántica, se ha buscado reconciliar y unificar ambas teorías (Becker, Becker y Schwarz, 2007). Así, "Del maridaje de la relatividad especial con la mecánica cuántica nació la teoría cuántica de campos" (Abdalla, 2005, p. 148). De este maridaje, nace la teoría de cuerdas que se lanza a construir "una teoría cuántica que unifique la descripción de la gravedad y la de otras fuerzas fundamentales de la naturaleza. En principio, esta tiene el potencial para proveer una completa comprensión de la física de partículas y de la cosmología” (Becker, Becker \& Schwarz, 2007, p. 3). La teoría de cuerdas apuntaría, entonces, a explicar los procesos microfísicos y macrofísicos, mediante una mecánica cuántica relativista, o sea, a través de una teoría cuántica de campos centrada en establecer una explicación cuántica de la gravedad (Becker, Becker \& Schwarz, 2007). 
En tanto la teoría de cuerdas explicaría la existencia de partículas (bosons y fermions) y fuerzas, sería preciso aceptar más dimensiones que las hasta hoy conocidas (Abdalla, 2005). Tendríamos entonces un universo donde pequeñas extradimensiones son asumibles (Randall, 2002).

La teoría de cuerdas y la cuestión de las dimensiones adicionales dan origen a la idea de las branas (membranas) que son objetos flotantes en un espacio dimensional más alto, que limitan la materia y las fuerzas, así como portan energía o tensión (Randall, 2002), creando una cosmología caracterizada por el estar viviendo en una rebanada (una membrana) espacio-temporal y multidimensional (Abdalla, 2005). Esto da origen a diversos y diferentes universos con otras leyes físicas y otras geometrías aún desconocidas (Randall, 2002), pero donde la gravedad no está confinada a una sola brana (Randall, 2005), sino que es más bien la gravedad la fuerza común que une estos universos (Abdalla, 2005).

Hasta aquí pareciera que todo sonara plausible y científico. No obstante, estas teorías son producto "más del pensamiento que del experimento" (Abdalla, 2005, p. 155), "Mezclando ciencia ficción y realidad" (Abdalla, 2005, p. 150) y convirtiéndose así en "un sueño distante" (Becker, Becker \& Schwarz, 2007, p. 3): "la teoría de cuerdas es hoy (...) in-probable" (Kaku, 2008, p. 297).

El problema con la física de la teoría de cuerdas es que "trata solo con modelos idealizados" (Plotnitsky, 2015, p. 559), donde un modelo es una colección de objetos ideales, matemáticos, o sea, un conjunto con todo lo que implica esto, de acuerdo con lo planteado en el apartado anterior.

La mediación entre algunas teorías "físicas" discutidas aquí y la teoría de conjuntos, en relación con la CF cuántica, se marca de manera muy clara en Kaku (2008) y, en la CF, con Rieder (2010).

\section{Fundamentos "físico-poéticos" de la CF cuántica}

A pesar de que Kaku piensa que "sin ciencia no hay ciencia ficción" (2008, p. x), él termina anulando su propia afirmación, al abordar elementos como la teoría de cuerdas (que es una mera conjetura) y los campos cuánticos para inventarse multiversos, dimensiones alternas, etc. (Kaku, 2008), cuestiones que pretenden romper el principio del tercero excluido de la lógica, por un mero acto volitivo. 
De igual modo, la única manera de sostener estos modelos que no existen (Frigg, 2010) es que nuevamente sea el conjunto $\varnothing$ el fundamento último de la propuesta de Kaku (2008).

No obstante, sobre esta línea de crear modelos (conjuntos), me interesa discutir algunas ideas de Rieder (2010) en tanto es él quien les da una forma más poética a las nociones ligadas a la teoría de conjuntos y sus implicaciones, en la CF.

Rieder (2010) establecería que la CF no tiene una esencia única, ni un origen determinado y que, ontológicamente, se debería ver como un modo de categorización, históricamente dado, desplegado por algún tipo de comunidad (Higgins, 2013).

Rieder (2010) parte, en su ensayo, de un gran error al evaluar la posición de Darko Suvin sobre la CF como formalista: "la literatura del extrañamiento cognitivo' de Darko Suvin ha sido de hecho, por mucho, la definición formal de más influencia" (Rieder, 2010, p. 193), cuando en realidad Angenot y Suvin han exteriorizado que una teoría válida de crítica es "simultáneamente formal y social" (1981, p. 122), luego es también histórica. Recordemos que, "Suvin no separa claramente en su definición de CF entre los aspectos ficticios, estilístico-formales y receptivos" (Spiegel, 2006, p. 20). En un esquema dialéctico, los elementos estilístico-formales refieren a veces a la forma en sí y en ocasiones al contenido, del mismo modo que los receptivos remiten a veces a lo intradiegético y otras veces a lo extradiegético (Alfaro-Vargas, 2014); no hay, por ende, una sustancialización de las categorías de forma y contenido en la CF.

Como, según Rieder (2010), Suvin habría planteado una definición formal(ista) de la CF, que la definiría de manera no-histórica e inmutable, entonces él nos dirá novedosamente que "la CF es histórica y mutable" (2010, p. 193), bajo la premisa de "la lógica superioridad de la aproximación histórica sobre la formal” (Rieder, 2010, p. 193).

Rieder remarca esta supuesta superioridad por medio de su énfasis en la teoría de conjuntos, particularmente, por la idea del conjunto difuso, el cual:

Más que estar determinado por un sencillo principio binario de inclusión o exclusión, está constituido por una pluralidad de operaciones. El conjunto difuso por tanto incluye elementos de cualquier rango de características y la 
afiliación al conjunto puede soportar muy diferentes niveles de intensidad, ya que algunos elementos tendrán la mayoría o todas las características requeridas mientras otros podrían tener solo una (Rieder, 2010, p. 194).

En términos lógicos, en un conjunto difuso no habría una correspondencia clara, directa y lineal entre la intensión lógica del conjunto (las características asociables a un elemento determinado, para poder pertenecer a un tal conjunto difuso) y la extensión (los posibles elementos de un tal conjunto difuso). Además, el conjunto difuso en tanto conjunto depende del axioma de existencia del que hablábamos arriba y, por consiguiente, está relacionado con el conjunto $\varnothing$.

Asimismo, la aplicación del conjunto difuso implicaría que "Si el pensamiento es un reflejo de la realidad en algún sentido, entonces la realidad estaría profundamente deshilvanada, plagada de huecos, vacíos, agujeros de gusano a otros universos" (Morton, 2014, p. 216) y la CF, como una forma de pensamiento (estético), compartiría las mismas características que el pensamiento en general; luego, todo vale: la CF habría devenido "una forma de discurso que directamente se compromete con el lenguaje y la cultura postmodernos" (Csicsery-Ronay, 1991, p. 388). Esto da finalmente la ilusión de que la competencia entre la CF y la fantasía está siendo ganada por esta última (Jameson, 2005), aunque, en el fondo, no es más que una imposición de los grupos neoliberales sobre la CF (Alfaro-Vargas, 2015), y no la simple convergencia de CF y postmodernidad (Peregrina, 2015).

De hecho, la cuestión de la teoría de conjuntos nos remite a la idea de sistema o de modelo. Las nociones sinonímicas, en este caso, de sistema y modelo se definen como "un conjunto de cosas y de relaciones entre ellas" (Castellani y Hafferty, 2009, p. 7), en cuanto la teoría de conjuntos nos permite "pensar, organizar y discutir la colección de objetos (conjuntos) y las relaciones que estos objetos (como conjuntos) tienen consigo mismos y con otros" (Castellani y Hafferty, 2009, p. 48).

Un modelo sería un artefacto, cuya validación es dada por el conjunto $\varnothing$ y, específicamente, por el axioma de existencia, si este reza que existe un conjunto; luego, un conjunto $\varnothing$ y, por ende, uno no-vacío también existirían. O sea, por un juego tautológico, se legitima prácticamente cualquier modelo que se quiera construir, ya que "Un modelo es una cosa artificial" (Oatley, 
2011, p. 3), que no implica "ninguna clase de corroboración empírica directa" (Callinicos, 2014, p. 153).

Así, un modelo es un conjunto cuyos elementos no se adhieren a tal conjunto (difuso) por compartir los mismos rasgos intensionales, sino por obra de la voluntad del agente, en cuanto la existencia de la nada (del conjunto $\varnothing$ ) le permite justificar y legitimar cualquier cosa. Esto nos permite pasar al segundo punto del planteamiento de Rieder (2010), es decir, la cuestión de la comunidad de práctica, el agente que Rieder establece para "manipular" el conjunto difuso que definiría los posibles modos de CF. Esta comunidad se definiría como un "nosotros" que "constituiría alguna clase de espejismo gramatical imputando una intencionalidad colectiva hacia un proceso sin un sujeto" (Rieder, 2010, p. 201), donde "la ciencia ficción podría ser tomada como el conjunto de objetos que las pertinentes comunidades de práctica señalan en común” (Rieder, 2010, p. 204; cursiva mía).

Las comunidades de práctica de la CF usarían "el género para dar activamente forma a su comprensión del mundo" (Rieder, 2010, p. 197), lo cual permitiría un mejor rango de posibilidades y determinaría los valores atribuidos a estas (Rieder, 2010). En una perspectiva totalmente fenomenológica, las comunidades de práctica se dirigirían (su intencionalidad) a establecer intensionalidades (con "s") pertinentes a su evento vivencial (Ereignis), lo cual, para Rieder, más que implicar una confusión, resultaría "de la variedad de motivos que las definiciones expresan y los muchos sentidos de intervenir en la producción, distribución y recepción del género que estas persiguen" (2010, p. 204). Entonces, cada comunidad de práctica podría crear su propio "modelo", su propia noción de CF, la cual cabe la posibilidad de que sea totalmente contradictoria en relación con el "modelo" de otra determinada comunidad de práctica y ambas serían igualmente válidas, en tanto responden a sus valores vivenciales y en cuanto también cada definición responde al proceso propio de producción, distribución y recepción de las mercancías circulantes como $\mathrm{CF}$.

En términos lógico-formales, lo que Rieder plantea es la anulación del principio de tercero excluido, que implica que $\mathrm{A}$ es $\mathrm{A}$ o no-A, pero nunca ambos a la vez. Es decir, las comunidades de práctica no seguirían los patrones supuestamente "rígidos" de la lógica formal, sino su elástica vivencialidad. Sin embargo, el sustento inicial de la reflexión de Rieder es 
la teoría de conjuntos, que es un cuerpo axiomático-deductivo basado en la lógica formal, la que da fundamento al conjunto $\varnothing$ y, por extensión, al conjunto difuso. En Rieder, hay una lógica formal que sirve cuando a él le interesa y que no funciona cuando a él no le interesa. Ergo, el planteamiento de Rieder es insostenible, ya que se autoanula.

No obstante, en el discurso pseudoacadémico y en el estético (en cine, por ejemplo) se emplea este enfoque basado en la teoría de conjuntos, la teoría de cuerdas, en propuestas como las de Kaku (2008) y poéticas como la de Rieder (2010), para darle un supuesto soporte epistemológico y operacionalidad estructurante a este conglomerado de elementos en la CF cuántica.

\section{Hacia una escatología no-teológica}

Aquí, nos centraremos en Interstellar (Nolan, 2014), debido a que esta muestra un desarrollo diegético más explícito y amplio de las características que se han dado para la CF cuántica y que nos permitirá profundizar todavía más en su conformación.

Interstellar exhibe algunos elementos propios de las perspectivas físicas ya mencionadas. Veamos, de esta película, el siguiente diálogo:

Amelia. -Pero de todas esas anomalías, la más significativa es esta: cerca de Saturno, un disturbio del tiempo-espacio.

Cooper. - ¿Es un agujero de gusano?

Amelia. - Apareció hace 48 años.

Cooper. - ¿Y lleva alguna parte?

Brand. -A otra galaxia (Nolan, 2014).

Este diálogo nos ofrece algunos conceptos ligados a versiones cosmológicas, relacionadas con las teorías físicas ya señaladas. Así, conceptos como espacio-tiempo, agujeros de gusano y la idea de que estos son pasadizos a otros mundos, ejemplifican la pretendida base científica de una película de CF cuántica como esta.

El siguiente diálogo refuerza todavía más lo expresado:

Amelia. -El tiempo es relativo, ¿de acuerdo? Este puede estirarse y estrecharse, pero no puede ir hacia atrás. Solo no puede hacerlo. La única cosa que 
puede moverse a través de las dimensiones, como el tiempo, es la gravedad (Nolan, 2014).

Hasta aquí, de acuerdo con las teorías que sostienen supuestamente el aspecto científico de Interstellar, hay coherencia, al establecer la gravedad como el único elemento que puede tener movimiento interdimensional. Mas, como la teoría de cuerdas es solo un modelo artificial sin base material-real, entonces se llega a un punto al cual el supuesto anclaje científico de esta película se cae, pierde veracidad (lo cual termina afectando la verosimilitud) y es obligada a recurrir a elementos absurdos, para poder continuar con la diégesis. Veamos este otro diálogo de Interstellar:

Amelia. -El amor es la única cosa que nosotros somos capaces de percibir, la cual trasciende las dimensiones de tiempo y espacio (Nolan, 2014).

Pero, ¿no era que solo la gravedad era capaz de tener una función interdimensional? ¿Es el amor de repente una fuerza física? Dentro de la propuesta de la teoría de cuerdas, sí, solo la gravedad tiene la propiedad de atravesar dimensiones; y no, el amor no es una fuerza física. Aquí lo que se da es el burdo recurso a una de las características del novum tecnocrático, o sea, "el énfasis en lo emocional en detrimento de lo racional" (Alfaro-Vargas, 2015, p. 13). Al no tener la teoría de cuerdas un arraigo material, Interstellar se sume en una nebulosa emocional, de un amor entre padre e hija.

Veamos cómo este recurso a lo emocional cierra una película que evidentemente representa el concepto del novum tecnocrático, en su versión de CF cuántica:

Hombre. -Entonces, ¿tienes alguna idea?

Murphy. -Un sentimiento. Te había contado acerca de mi fantasma. Mi papá pensó que yo lo llamaba un fantasma porque estaba asustada. Pero yo nunca estuve asustada por él. Lo llamé fantasma porque yo sentía... yo lo sentía como una persona. Él estaba tratando de decirme algo. Si hay una respuesta aquí en la Tierra, es allá... de alguna manera, en aquel cuarto. Entonces tengo que encontrarla (Nolan, 2014). 
Como se observa aquí, en este tipo de trama es más importante el sentimiento que lo racional. Se ha pasado de conceptos como gravedad, agujeros de gusano y espacio-tiempo, a nociones como amor y sentimientos. El siguiente diálogo termina de completar esta idea:

Cooper. -Por esto estoy yo aquí. Voy a encontrar un modo de hablar con Murphy justo como encontré este momento.

TARS. - ¿Cómo, Cooper?

Cooper. -El amor, TARS, el amor (Nolan, 2014).

Finalmente, la Tierra es salvada por el amor de un padre que, con su voluntad, rompe el destino distópico con el que inicia Interstellar, teniendo como resultado un conjunto con elementos que no comparten, en general, la intensión (o sea, algún tipo de característica en común) y que, por ende, contienen una extensión basada en elementos físico-teóricos y emocionales. Luego, tendríamos el conjunto Interstellar = \{teoría de cuerdas, campos cuánticos, gravedad, viajes a la velocidad de la luz, agujeros de gusano, amor, sentimientos, $\varnothing\}$, donde si este conjunto $\varnothing$ existe, entonces todo conjunto que lo contenga también existiría. Con este tipo de fundamento opera Interstellar, como una expresión novum-tecnocrática de la CF cuántica. El problema es que esto es solo lenguaje matemático, derivado y anclado en el natural y el lenguaje no crea realidad.

No obstante, hay dos cuestiones en el nivel ideológico que es necesario resaltar. La primera tiene que ver con la teoría de conjuntos como forma de eliminar o evitar los problemas con la noción de totalidad (Lacan, 1966), que finalmente fundamenta ontoepistemológicamente la CF cuántica. En segundo lugar, está el asunto de la estructuración de una escatología no-teológica, como un medio de control social.

La ontología de la teoría de conjuntos se diferencia de la noción de totalidad, en que esta teoría es pura forma, es decir, es un cuerpo axiomático ligado a principios de la lógica formal, pero sin contenido real, material. El conjunto $\varnothing$ es solamente una idea.

En cambio, la noción de totalidad es la unidad, entre otras cosas, de forma y contenido, o sea, la estructura lógica se ve siempre confrontada y superada (aufgehoben) por un contenido que está, a diferencia de la rigidez 
propia de un concepto, en transformación y que supone un sujeto que no solo piensa, sino que actúa sobre su objeto. Aquí la noción de conjunto $\varnothing$ es superada (aufgehoben) por lo concreto.

Así, la teoría de conjuntos como elemento ontoepistemológico estructurante de la CF cuántica genera mercancías que (en tanto forma estética) evade, oculta y tergiversa el contexto de producción, creando diégesis futuristas que no asumen hechos como la crisis sistémico-económica, la cual le ha quitado la preponderancia mundial a Estados Unidos o las altas tasas de desempleo, etc., que ese país sufre; todos estos elementos constituidos como el contenido que la forma estética de la CF cuántica -en Interstellar, como ejemplo-niega, ofreciendo a cambio una escatología no-teológica que no es más que la expresión mayormente nihilista del conjunto $\varnothing$.

La escatología no-teológica se da mediante una serie de conjeturas ligadas a la teoría de conjuntos, donde las ideas vinculadas a la existencia de universos paralelos y múltiples dimensiones, dentro de un campo cuántico, fundamentan una doctrina de promesas futuras, que "reconfortan" en el aquí y ahora.

Kaku (2008), de hecho, expresa que una mujer podría estar en diferentes estados en los diversos universos: embarazada, anciana, etc. En este marco, la CF cuántica, como estructura nóvico-tecnocrática, ofrece hasta la inmortalidad, como en Terminator Genisys (Taylor, 2015), en la cual Kyle viene de una dimensión, donde las máquinas han casi exterminado la humanidad, pero que al viajar en el tiempo accede a un nexus que le permite tener recuerdos de un mundo paralelo donde es posible evitar el surgimiento de la aplicación Skynet y, por ende, en esa otra dimensión, quienes en una dimensión murieron vivirían el fenómeno de la vida eterna hecho "realidad". Se produce así una trascendencia escatológica sin dios alguno, pero sí con cuerdas y multidimensiones, muy apropiada al ofrecer un más allá interpretable de acuerdo con los credos propios de cada "comunidad de práctica" que consuma esta CF cuántica.

Del mismo modo, hay en esta escatología un velado quietismo político, ya que, si eres obrero explotado en esta dimensión, no es preciso buscar la transformación social, debido a que en "otra” dimensión quizás eres capitalista. El estar entonces en una clase social baja o en una alta sería solamente 
la expresión de un azar cuántico, probabilístico y no de la apropiación o no de la plusvalía.

En la CF cuántica, el sujeto histórico se reduce a una vulgar noción antropológica donde existe un sesgado voluntarismo, como ideología, que lo desmiembra y lo representa como un despojo de lo humano, ya que esta antropología de la CF cuántica (su cronotopo) tiene en sí un ser humano encasillado en lo emocional. En Interstellar, no es lo racional el principio activo de una transformación que une sujeto y objeto, ser y pensamiento; ni tampoco es, en consecuencia, el ser humano total el que actúa, sino una piltrafa humana de amor y sentimientos que, según esta $C F$, es más fuerte que las mismas leyes físicas.

\section{Conclusión}

En el fenómeno del novum tecnocrático, existe la CF cuántica, caracterizada por ligarse a los planteamientos de algunas teorías físicas, como la teoría de cuerdas, teniendo como fundamento ontoepistemológico la teoría de conjuntos; donde la función de esta CF cuántica es elaborar una escatología no-teológica y promover, en algunos casos, una concepción burdamente voluntarista.

En relación con el novum tecnocrático, la CF cuántica revela algunas nuevas características de tal novum no descritas por Alfaro-Vargas (2015). Así, junto a elementos como: el extrañamiento no-cognitivo, el énfasis sobre lo emocional, la función pedagógico-ideológica en la estimulación de la enseñanza-aprendizaje de la matemática y las ciencias, el sistema de estrellas (en cine), el efecto de espectacularidad y su función como mecanismo ideológico de control social (Alfaro-Vargas, 2015) tenemos también, consecuentemente, una base ontoepistemológica fundamentada en la teoría de conjuntos, un voluntarismo individualista y una escatología no-teológica.

Asimismo, es importante resaltar el hecho de que la CF del novum tecnocrático evidencia un double bind con respecto al principio de tercero excluido. Es decir, se plantea de fondo una CF que es ella misma y fantasía a la vez, o sea, es CF y su negación en un mismo momento, lo cual es irracional y, por tanto, irreal. No obstante, absurdamente, el fundamento ontoepistemológico es un cuerpo lógico-deductivo (la teoría de conjuntos) 
amparado en la lógica aristotélica. El novum tecnocrático y la CF cuántica son fenómenos ideológicos sumamente contradictorios, que conllevan su propia anulación, son fenómenos nihilistas e irracionales, debido a su relación con el conjunto $\varnothing$, con la nada, con lo real lacaniano.

\section{Referencias}

Abdalla, E. (2005). Teoria quântica da gravitação: Cordas e teoria M. Revista Brasileira de Ensino de Física, 27(1), 147-155. Recuperado de https://doi.org/10.1590/S180611172005000100017

Alber, J. (2016). Unnatural Narratives. Impossible Worlds in Fiction and Drama. Lincoln and London: University of Nebraska Press.

Alber, J., Iversen, S., Nielsen, H. S. \& Richardson, B. (2013). What Really Is Unnatural Narratology? Story Worlds: A Journal of Narrative Studies, 5, 101-118.

Alfaro-Vargas, R. (2014). Marxismo y ciencia ficción. Praxis, 72, 81-97.

Alfaro-Vargas, R. (2015). El novum tecnocrático. Hélice, II (5), 6-20.

Alfaro-Vargas, R. (2016). Totalidad y teoría de conjuntos. Revista de Ciencias Sociales, II(152), 133-144.

Angenot, M. \& Suvin, D. (1981). Thèses sur la «sociologie» de la littérature. Littérature, 44, 117-127. Recuperado de https://do.org/10.3406/litt.1981.1365

Badiou, A. (2006). Logiques des mondes. L'Être et l'événement, 2. Paris: Éditions du Seuil.

Becker, K., Becker, M. \& Schwarz, J. H. (2007). String Theory and M-Theory. A Modern Introduction. New York: Cambridge University Press.

Burhanuddin, B. (2015). Badiou's Being and Event and the Mathematics of Set Theory. London and New York: Bloomsbury.

Callinicos, A. (2014). Deciphering Capital. Marx's Capital and its Destiny. London: Bookmarks Publications.

Castellani, B. \& Hafferty, F. W. (2009). Sociology and Complexity Science. A New Field of Inquiry. Heidelberg: Springer. Recuperado de https://doi.org/10.1007/978-3-540-88462-0

Csicsery-Ronay, I. Jr. (1991). The SF of Theory: Baudrillard and Haraway. Science Fiction Studies, 18(3), 387-404.

Frigg, R. (2010). Models and Fiction. Synthese, 172, 251-268. https://doi.org/10.1007/ s11229-009-9505-0

García-Martínez, A. \& González, A. M. (2016). Emotional Culture and TV Narratives. En Alberto N. García (Ed.), Emotions in Contemporary TV Series (1325). London and New York: Palgrave McMillan. Recuperado de https://doi. org/10.1007/978-1-137-56885-4_2

Gironi, F. (2015). Naturalising Badiou. Mathematical Ontology and Structural Realism. London and New York: Palgrave Macmillan.

Han, B-C. (2014). Psicopolítica. Neoliberalismo y nuevas técnicas de poder. Barcelona: Herder. 
Higgins, D. M. (2013). Coded Transmissions. Gender and Genre Reception in The Matrix. En B. Attebery \& Veronica Hollinger (Eds.), Parabolas of Science Fiction. (143-160). Middletown, Connecticut: Wesleyan University Press.

Jameson, F. (2005). Archaeologies of the Future. The Desire Called Utopia and Other Science Fictions. London and New York: Verso.

Kaku, M. (2008). Physics of the Impossible: A Scientific Exploration into the World of Phasers, Force Fields, Teleportation, and Time Travel. New York: Doubleday.

Lacan, J. (1966). Écrits. Paris: Éditions du Seuil.

Lefebvre, H. (2016). Metaphilosophy. London and New York: Verso.

May, A. (2017). Pseudoscience and Science Fiction. Switzerland: Springer. Recuperado de https://doi.org/10.1007/978-3-319-42605-1

Morton, T. (2014). Pandora's Box. Avatar, Ecology, Thought. En G. Canavan \& K. S. Robinson (Eds.), Green Planets. Science Fiction and Ecology. (206-225). Middletown, Connecticut: Wesleyan University Press.

Nolan, C. (Director). (2014). Interstellar. Warner Bros., Syncopy, Paramount Pictures, Legendary Pictures and Lynda Obst Productions.

Oatley, K. (2011). Such Stuff as Dreams. The Psychology of Fiction. Malden (MA)-Oxford: Recuperado de Wiley-Blackwell. https://doi.org/10.1002/9781119970910

Peregrina, M. (2015). La ciencia ficción y la narrativa posmoderna: hacia la convergencia. Alambique: Revista académica de ciencia ficción y fantasía, 3(1). Recuperado de: http:// scholarcommons.usf.edu/alambique/vol3/iss1/2

Plotnitsky, A. (2015). Reality, Causality, and Probability, from Quantum Mechanics to Quantum Field Theory. En T. M. Nieuwenhuizen, C. Pombo, C. Furtado, A. Yu Khrennikov, I. A. Pedrosa \& V. Špi ka (Eds.) Quantum Foundations and Open Quantum Systems. Lecture Notes of the Advanced School. (521-603). Singapur: World Scientific Publishing.

Randall, L. (2002). Extra Dimensions and Warped Geometries. Science, 296 (24), 1422 1427. Recuperado de https://doi.org/10.1126/science.1072567

Randall, L. (2005). Warped Passages. Unraveling the Mysteries of the Universe's Hidden Dimensions. Toronto and Pymble (Australia): HarperCollins e-books.

Rieder, J. (2010). On Defining SF, or Not: Genre Theory, SF, and History. Science Fiction Studies, 37(2), 191-209.

Saldías R., G. (2016). A propósito de «El novum tecnocrático» de Roy Alfaro Vargas. Hélice, III (6), 6-12.

Spiegel, S. (2006). Der Begriff der Verfremdung in der Science-Fiction-Theorie. Ein Klärungsversuch. Quarber Merkur. Franz Rottensteiners Literaturzeitschrift für Science Fiction und Phantastik, 103/104, 13-40.

Stock, K. (2016). Imagination and Fiction. En A. Kind (Ed.), The Routledge Handbook of Philosophy of Imagination (204-216). London and New York: Routledge.

Taylor, A. (Director). (2015). Terminator Genisys. Paramount Pictures, Annapurna Pictures and Skydance Productions. 